\title{
Questionário de Vivências Acadêmicas (QVA-R): avaliação de estudantes de medicina em um curso com currículo inovador
}

\author{
Diego Roberto Lima dos Anjos \\ Rinaldo Henrique Aguilar-da-Silva
}

Resumo: Este artigo analisa o impacto do currículo inovador na adaptação dos estudantes de medicina, propondo-se verificar os fatores que interferem na vivência acadêmica. Utilizamos o Questionário de Vivências Acadêmicas na sua versão reduzida (QVA-r), com escala de Likert de 5 pontos. Participaram 90 estudantes de medicina, 40 da $1^{\text {a }}$ série e 40 da $3^{\text {a }}$ série. Em relação ao gênero $60 \%(n=48)$ foram do sexo feminino com (QVA-r total 3,7) e $40 \%(\mathrm{n}=32$ ) do masculino com (QVA-r total 3,8). Os participantes que exerciam atividade remunerada apresentaram média inferior na dimensão estudo $(M=3,3)$ em relação aos que não exerciam nenhuma atividade remunerada $(\mathrm{M}=3,6)$. Observamos que a dimensão carreira exerce um papel importante na adaptação dos estudantes $(M=4,2)$ para ambas as séries. Foram identificadas diferenças referentes à idade, evidenciando que os estudantes com maior média demonstraram melhor adaptação à instituição. Constatamos que as mulheres apresentam melhor adaptação ao método de ensino e os homens demonstram melhor adaptação global à instituição. Concluímos que o QVA-r demonstrou ser um bom instrumento de avaliação da adaptação dos estudantes frente à implantação de currículos inovadores no curso médico.

Palavras-chave: Educação médica. Diretrizes curriculares nacionais. Adaptação à universidade.

\section{Academic Experiences Questionnaire (QVA-R): \\ medical students' evaluation in an innovative resume course}

Abstract: This article analyses the innovative resume impact on medical students' adjustment process with a purpose of verifying the factors which interfere on academic experience. A reduced version of Academic Experience Questionnaire was used (QVA-r), in a five score Likert scale. Ninety medical students took part, where forty of them were from the first grade and forty from the third. Ten students were excluded due to uncompleted questionnaire and/or consent form. $60 \%(\mathrm{n}=48)$ were female (QVA-r 3,7 overall value) and $40 \%(\mathrm{n}=32)$ male (QVA-r 3,8 overall value). The participants who pursued gainful employment presented a lower average on a study dimension $(M=3,3)$ than the ones with no gainful employment $(\mathrm{M}=3,6)$. It was observed in both grades that a career dimension plays an important role on students adjustment $(M=4,2)$. Differences related to age were identified evincing that students with a largest average showed the best adjustment to the institution. Women were determined with the best adjustment to the teaching method and men with the best global adjustment to the institution. QVA-r has proved to be a good student's adjustment assessment tool forward the innovative resume implantation on medical course.

Key words: Medical education. National curriculum guidelines. College adaptation. 


\section{Introdução}

As Diretrizes Curriculares Nacionais (DCN) dos Cursos de Graduação da área da saúde constituem-se em uma alteração no processo de Educação Superior ao romper o modelo flexneriano, biomédico e curativo e assumir um modelo voltado para saúde-doença com atuação em todos os níveis de atenção à saúde. Este modelo curricular parte de uma dimensão individual para a coletiva, libertando-se dos currículos rígidos compostos por disciplinas fragmentadas com priorização das atividades teóricas, para currículos flexíveis e modulares visando aquisição de competências profissionais, os quais exigem mudanças nas metodologias de aprendizagem (BRASIL, 2014; STELLA, 2007).

No entanto, o grande desafio das DCN é a formação de recursos humanos em saúde nos cursos de graduação em Medicina, por meio de médicos com formação generalista, humanista com caráter crítico reflexivo, pautado em princípios éticos e no processo de saúde-doença com atuação nos três níveis de atenção (ALMEIDA et al., 2005).

No Brasil, e no mundo, há inúmeros fóruns que discutem os conceitos que envolvem a mudança da formação médica e de outros profissionais da área da saúde. Considerando que a educação superior não apresenta só o objetivo de instrução e capacitação profissional, mas também almeja a formação de profissionais capazes de desempenhar habilidades peculiares à sociedade em que estão inseridos (GOMES; REGO, 2011).

Sendo assim a universidade tem o papel de favorecer a capacitação técnica e o desenvolvimento das habilidades psicomotoras, cognitivas e afetivas, além da ética e do compromisso com a cidadania. Deve abordar aspectos como a integralidade do cuidado em saúde e aprimorar os conceitos da humanização (SOARES; POUBEL; MELLO, 2009).

De acordo com Soares, Poubel e Mello (2009), essas transformações acabam gerando a necessidade de ajustes para atender as novas demandas educacionais, e as universidades assumem um papel não só de se preocupar com a aquisição de conhecimentos, mas também de comprometer-se com estratégias de adaptação às novas situações. Isto se dá por meio do desenvolvimento das competências acadêmicas, cognitivas e sociais, garantindo o sucesso do estudante desde seu ingresso na universidade.

Nas últimas décadas o ensino superior passou por acentuadas transformações para atender às demandas da sociedade. Diante dessas metamorfoses, as universidades passaram a buscar uma nova organização, ressignificando a maneira de a sociedade produzir e de transmitir seus valores, a fim de propor- 
cionar melhorias nas condições humanas diante de suas múltiplas dimensões (CUNHA; CARRILLO, 2005).

Assim, em diversas partes do mundo se fez necessário a reorientação para a formação de profissionais com perfis e capacidades distintas das produzidas pelo modelo hegemônico de formação (FEUERWERKER; LIMA, 2002).

A mudança do ensino médio para o ensino superior, para a maioria dos estudantes, pode ser vista de forma positiva e emocionante, em virtude de possibilitar realizações de projetos de carreira, profissional e pessoal. Esta fase também proporciona maior liberdade parental, novos vínculos e relacionamentos interpessoais além das responsabilidades acadêmicas, pessoais e sociais que acabam contribuindo para o desenvolvimento da sua identidade e autonomia (ALMEIDA, 2007; ALMEIDA; CRUZ, 2010).

A entrada na Universidade é um momento distinto na vida do estudante que gera oportunidades e novas experiências de aprendizagem e de desenvolvimento psicossocial. Por outro lado, esse processo de transição também pode ocasionar períodos estressantes e ansiogênicos para os estudantes que se encontram em processo de adaptação ao novo modelo acadêmico (FRIEDLANDER et al., 2007; MAZÉ; VERLHIAC, 2013).

Segundo a literatura, esse processo de transição do ensino médio para ensino superior pode ser mais simples para uns estudantes do que para outros. Porém, para se alcançar o sucesso nesse processo é necessário considerar os fatores acadêmicos pregressos, capacidades intelectuais, além de fatores interpessoais, pessoais, de personalidade e otimismo (FELDT, 2011).

Esta importância da transição do Ensino Médio para o Ensino Superior vem despertando a atenção de diversos estudiosos, envolvidos com esse contexto da adaptação e integração acadêmica (MOGNON; SANTOS, 2013).

Para minimizar os possíveis problemas referentes a este processo é importante que as instituições de ensino implementem programas de intervenção psicopedagógicas, com o intuito de facilitar a adaptação do estudante e com isso reduzir os transtornos da transição do ensino médio para o ensino superior nesses indivíduos. Dessa forma a instituição pode desenvolver inúmeras atividades que visam o desenvolvimento pessoal do mesmo, capacitando-o tanto para as atividades acadêmicas como para o desenvolvimento da sua personalidade (CUNHA, 2004; VILLAR, 2003).

De acordo com Soares, Poubel e Mello (2009), no primeiro ano da universidade é fundamental uma atenção especial à chegada dos estudantes, consequentemente, na sua adaptação à instituição. Essa atenção deve ser redobrada aos estudantes que se sentem menos preparados para o processo de mudança e/ou mais fragilizados em termos psicossociais. 
Estudos demonstram que mais da metade dos estudantes mencionam algum tipo de dificuldade nesse momento de transição acadêmica. Isso implica em altas taxas de insucesso e de abandono dos estudantes nos primeiros anos do curso, sobretudo no primeiro ano da universidade (ALMEIDA; SOARES; FERREIRA, 2002).

Para Santos e Almeida (2001), o processo de adaptação está relacionado principalmente ao primeiro ano, que estabelece um preditor importante de perseverança e de sucesso do estudante no decorrer de sua vida acadêmica, bem como do real aproveitamento das novas oportunidades de desenvolvimento psicossocial. Ainda assim, para que os estudantes tenham um desenvolvimento satisfatório, é necessário um ambiente acadêmico que contenha um nível adequado tanto de desafio, como de apoio. Porém esse equilíbrio nem sempre é fácil de alcançar e com isto as características individuais assumem um papel mediador neste processo.

Em um mundo extremamente competitivo, a Universidade precisa preocupar-se com o estudante, promovendo condições para o seu desenvolvimento integral, tentando desenvolver suas potencialidades ao máximo. Assim, o estudante poderá atingir seu nível de excelência pessoal e estar preparado para um papel atuante na sociedade (SARRIERA et al., 2012).

Frente a esta realidade é fundamental para o sucesso da implantação curricular, avaliar e entender como os estudantes se adaptam aos novos processos de formação durante a graduação médica. O objetivo deste estudo é avaliar o impacto de um currículo inovador na adaptação dos estudantes, junto ao curso médico e identificar os fatores que interferem positivamente e negativamente para a adaptação dos estudantes.

\section{Procedimentos metodológicos}

No trabalho que originou este artigo, realizou-se uma pesquisa cuja abordagem é de enfoque quantitativo, com ênfase na primeira e terceira do curso de medicina, sendo um estudo de campo de caráter exploratório. A seleção da amostra foi não probabilística, com indivíduos voluntários; a coleta de dados foi realizada por meio de um questionário.

A amostra, neste caso, é um subgrupo da população de estudantes de uma Faculdade do interior paulista. Assim, a aplicação do questionário foi realizada em contexto de sala de tutoria, e durante as conferências, em regime presencial com contato direto com os estudantes. 
Neste momento foi possível explicar e informar os mesmos acerca dos objetivos da investigação, a importância da sua colaboração, bem como esclarecer as dúvidas que estes pudessem apresentar referente ao preenchimento.

\subsection{Local de realização/ Estrutura curricular}

O campo de pesquisa foi a Faculdade de Medicina de Marília (Famema). A escolha deste cenário deu-se pelo fato da instituição ser a pioneira na utilização de metodologias ativas de ensino e aprendizagem, além de possuir um currículo inovador no curso de Medicina.

A estrutura curricular que se encontra organizada por séries nas seguintes unidades: Unidade de Prática Profissional (UPP), Unidade Educacional Sistematizada (UES) e Unidade de Educacional Eletiva (UEE), esta desenvolvida a partir da $2^{\mathrm{a}}$ série (FAMEMA, 2012).

As intencionalidades da UPP e da UES são de proporcionar o desenvolvimento de recursos cognitivos, afetivos e psicomotores, articulados com tarefas que contribuam para a identificação das necessidades de saúde do indivíduo e do seu meio social e coletivo para compreensão da gestão do trabalho em saúde (FAMEMA, 2012).

A UPP está organizada em ciclos pedagógicos nas duas primeiras séries e desenvolvida nas Unidades de Saúde da Família onde são utilizadas metodologias da Problematização com enfoque na atenção primária. Na terceira série os estudantes são inseridos no ambiente hospitalar e os cenários são: enfermarias de pediatria, ginecologia e obstetrícia, clínica médica, cirurgia e psiquiatria.

É importante salientar que os estudantes estão incluídos nos cenários reais de práticas (UPP-1/UPP-2) desde o primeiro semestre da graduação, iniciando no $1^{\mathrm{a}}$ e $2^{\mathrm{a}}$ séries nas Unidades de Saúde da Família. Na $3^{\mathrm{a}}$ série ocorre a inserção nos cenários hospitalares, os quais correspondem à Unidade de Prática Profissional (UPP-3) (FAMEMA, 2012).

\subsection{Participantes}

Foi definida uma amostra de conveniência composta por 90 discentes que aceitaram participar voluntariamente, sendo 40 estudantes da $1^{\text {a }}$ série e 40 da $3^{\mathrm{a}}$ série. Foram excluídos 10 estudantes que não preencheram todas as questões e/ou termo de consentimento livre esclarecido.

Os participantes que compõem a população deste estudo cumpriram os seguintes critérios de inclusão: estar regularmente matriculado no curso de Medicina da Famema, cursando a primeira e a terceira série dos períodos letivos no ano de 2014. As idades variaram entre 17 a 31 anos com média de 21,95 anos. 


\subsection{Instrumento}

Para a coleta de informações foi utilizado o Questionário de Vivências Acadêmicas, versão reduzida - QVA-r. O Questionário de Vivências Acadêmicas, na sua versão original - QVA; idealizado por Almeida, Soares e Ferreira (2002), é um instrumento de autorrelato constituído por 170 itens de formato Likert, distribuído por 17 subescalas que procuram avaliar dimensões pessoais, relacionais e institucionais da adaptação dos estudantes ao contexto universitário.

O QVA-r, trata-se de um instrumento de autorrelato preenchido pelos participantes sem auxílio do pesquisador, constituído na versão brasileira por 55 itens, que se referem ao pensamento e sentimento dos estudantes em relação à universidade.

O instrumento no Brasil foi adaptado por Granado et al. (2005), verificando as evidências de validade relativas à estrutura interna dos itens. Os valores para a consistência interna mostraram-se adequados, com alfas de Cronbach para as dimensões variando de 0,71 a 0,91 e para a escala geral de 0,88 .

$\mathrm{O}$ instrumento de coleta de dados consiste em uma escala com pontuações do tipo escala de Likert de 5 pontos, variando de 1 (nada a ver comigo) a 5 (tudo a ver comigo). Na sua versão reduzida apresenta cinco dimensões (quadro 1), relativas às áreas de adaptação acadêmica: pessoal (14 itens), interpessoal (12 itens), carreira (12 itens), estudo (9 itens) e institucional (8 itens).

\section{Quadro 1 - Descrição das dimensões do QVA-r}

\begin{tabular}{|l|l|}
\hline Dimensão Pessoal & Itens \\
\hline $\begin{array}{l}\text { Refere-se ao bem-estar físico e psicológico; abordam aspectos como } \\
\text { o equilíbrio emocional, a estabilidade afetiva, o otimismo, a tomada de } \\
\text { decisões e a autoconfiança. }\end{array}$ & $\begin{array}{l}4,6,9,11,13,17,20,23, \\
25,27,34,40,47,50 .\end{array}$ \\
\hline Dimensão Interpessoal & \\
\hline $\begin{array}{l}\text { Refere-se às relações com os colegas e a competências de relacionamento } \\
\text { em situações de maior intimidade; inclui o estabelecimento de amizades, } \\
\text { a procura de ajuda. }\end{array}$ & $\begin{array}{l}1,18,22,24,26,29,31,33, \\
35,37,38,54 .\end{array}$ \\
\hline Dimensão Carreira & \\
\hline $\begin{array}{l}\text { Refere-se a sentimentos relacionados com o curso frequentado e } \\
\text { perspectivas de carreira; inclui a satisfação com o curso e percepção de } \\
\text { competências para o curso. }\end{array}$ & $\begin{array}{l}2,5,7,8,14,19,21,32, \\
46,49,51,55 .\end{array}$ \\
\hline $\begin{array}{l}\text { Dimensão Estudo } \\
\text { Refere-se a hábitos de estudo e à gestão de tempo; inclui as rotinas } \\
\text { de estudo, o planejamento do tempo, a utilização de recursos de } \\
\text { aprendizagem, a preparação para os testes. }\end{array}$ & $\begin{array}{l}10,28,30,36,39,42,44, \\
48,52 .\end{array}$ \\
\hline $\begin{array}{l}\text { Dimensão Institucional } \\
\text { Refere-se à apreciação da instituição de ensino frequentada; inclui os } \\
\text { sentimentos relacionados à instituição, o desejo de permanecer ou mudar } \\
\text { de instituição, conhecimento e apreciação das infraestruturas }\end{array}$ & $\begin{array}{l}3,12,15,16,41,43, \\
45,53 .\end{array}$ \\
\hline
\end{tabular}

Fonte: ALMEIDA, Leandro Santos; SOARES, Ana Paula C.; FERREIRA, Joaquim Armando. Questionário de vivências acadêmicas (QVA-r): Avaliação do ajustamento dos estudantes universitários. Avaliação Psicológica, Coimbra, v. 1, n. 2, p. 81-93, 2002. 


\subsection{Análise dos dados}

Para responder ao objetivo do estudo, além de técnicas básicas de análise exploratória de dados como média, mediana, desvio padrão, frequência absoluta e relativa, foi utilizado o coeficiente de correlação não paramétrico de Spearman. O software utilizado para a realização da análise estatística foi o The SAS System versão 9.0.

A correlação de Spearman serviu para avaliar a existência e intensidade da associação entre as 5 dimensões. O coeficiente de correlação de Spearman varia entre - 1 e 1 . O sinal indica a direção da correlação (negativa ou positiva) enquanto que o valor indica a magnitude.

Quanto mais perto de 1 mais forte é o nível de associação linear entre as variáveis.

De acordo com Dancey e Reidy (2006), que propõem uma classificação para análise da correlação de Spearman: $\mathrm{r}=0,10$ até 0,30 (fraco); $\mathrm{r}=0,40$ até 0,60 (moderado); $\mathrm{r}=0,70$ até 1 (forte) a seguinte classificação no que diz respeito à magnitude do coeficiente

Todos os testes de hipóteses desenvolvidos nesse trabalho consideraram uma significância de $5 \%$, isto é, a hipótese nula foi rejeitada quando p-valor foi menor ou igual que 0,05 .

\subsection{Aspectos éticos}

De acordo com a Resolução $n^{\circ} 466$, de 12 de dezembro de 2012, que regula a ética em pesquisa envolvendo seres humanos no Brasil, este estudo passou por apreciação do Comitê de Ética em Pesquisa Envolvendo Seres Humanos (CEP) da FAMEMA. Neste momento o estudo recebeu o número do Certificado de Apresentação para Apreciação Ética (CAAE)nº 25298313.1.0000.5413.

\section{Resultados}

Na tabela 1 são apresentadas as variáveis: sexo, faixa etária, série e exercício de atividade remunerada.

As idades variaram entre 17 e 31 anos (Média $=21,95$ anos), sendo 40\% (n $=32)$ do sexo masculino e $60 \%(\mathrm{n}=48)$ do sexo feminino.

Houve um predomínio dos estudantes na faixa etária de 21 a 25 anos, $60 \%$ $(\mathrm{n}=48)$. Na faixa etária de até 20 anos encontramos um percentual de $32,5 \%$ $(\mathrm{n}=26)$ da amostra. O percentual de estudantes com idade superior a 25 anos foi de $7,5 \%(n=6)$. 
Destacamos que $87,5 \%(n=70)$ dos estudantes afirmaram não exercer atividades remuneradas concomitantemente ao estudo e $12,5 \%(n=10)$ informaram exercer atividade remunerada sem horário fixo.

Tabela 1 - Caracterização dos estudantes em relação à frequência das variáveis: sexo, faixa etária, exercício de atividade remunerada e série do curso.

\begin{tabular}{lllc}
\hline Variável & Categoria & N & \% \\
\hline Sexo & Feminino & 48 & 60 \\
& Masculino & 32 & 40 \\
\hline Faixa etária & De até 20 anos & 26 & 32,5 \\
& De 21 a 25 & 48 & 60 \\
& Maior que 25 anos & 6 & 7,5 \\
\hline Exerce atividade remunerada & Não & 70 & 87,5 \\
& Sim & 10 & 12,5 \\
\hline Série & $1^{\text {a }}$ série & 40 & 50 \\
& $3^{\text {a }}$ série & 40 & 50 \\
\hline
\end{tabular}

Na tabela 2 observamos, os resultados da média, mediana e desvio padrão das 5 dimensões do QVA-r da $1^{\text {a }}$ série, onde identificamos que todas apresentaram scores maiores que a pontuação média, ou seja, valores de média acima de 3. No entanto, a dimensão que apresentou o melhor índice de adaptação foi a dimensão carreira $(M=4,2)$, seguida pela dimensão estudo $(M=3,6)$, revelando que ambas dimensões são bastante presentes nas vivências acadêmicas dos estudantes da $1^{\circ}$ série.

Por outro lado, a dimensão Institucional demonstrou menor índice de adaptação $(\mathrm{M}=3,2)$ na $1^{\mathrm{a}}$ série.

Tabela 2 - Análise da média, mediana e desvio padrão das 5 dimensões do QVA-r da $1^{\mathrm{a}}$ série

\begin{tabular}{llll}
\hline Dimensão & Média & Mediana & Desvio padrão \\
\hline Pessoal & 3,3 & 3 & 0,65 \\
Interpessoal & 3,4 & 3 & 0,42 \\
Carreira & 4,2 & 4 & 0,41 \\
Estudo & 3,6 & 4 & 0,57 \\
Institucional & 3,2 & 3 & 0,59 \\
\hline
\end{tabular}


Na tabela 2.1, temos os resultados da média, mediana e desvio padrão das 5 dimensões do QVA-r da $3^{\mathrm{a}}$ série. Assim como na $1^{\mathrm{a}}$ série a dimensão carreira apresentou média superior $(M=4,2)$, revelando ser a dimensão com o melhor fator de adaptação.

Em seguida temos a dimensão estudo $(\mathrm{M}=3,7)$ evidenciando que os estudantes da $3^{\mathrm{a}}$ série apresentam melhor tendência de adaptação ao método de ensino utilizado pela faculdade.

No entanto, a dimensão institucional $(\mathrm{M}=3,4)$ exibiu a pior média entre as dimensões, configurand o-se como o menor índice de adaptação dos estudantes.

Tabela 2.1 - Análise da média, mediana e desvio padrão das 5 dimensões do QVA-r da $3{ }^{\text {a }}$ série

\begin{tabular}{llll}
\hline Dimensão & Média & Mediana & Desvio padrão \\
\hline Pessoal & 3,7 & 4 & 0,63 \\
Interpessoal & 3,6 & 4 & 0,55 \\
Carreira & 4,2 & 4 & 0,51 \\
Estudo & 3,6 & 4 & 0,61 \\
Institucional & 3,4 & 4 & 0,52 \\
\hline
\end{tabular}

Na Tabela 3, apresentamos a média das variáveis: sexo, faixa etária, exercício de atividade remunerada e série para cada uma das seguintes dimensões. Constatamos que os estudantes do sexo masculino demonstraram melhor adaptação global ao curso em relação às estudantes do sexo feminino.

Evidenciamos que na faixa etária de 21 a 25 anos os estudantes apresentaram melhor índice de adaptação global à instituição.

Já em relação à série do curso, observamos uma tendência de aumento das médias na $3^{\text {a }}$ série para as dimensões pessoal, interpessoal e institucional, o que pode demonstrar um melhor nível de adaptação. 
Tabela 3 - Avaliação das dimensões do QVA-r de acordo com as variáveis: sexo, faixa etária, exercício de atividade remunerada e série do curso

\begin{tabular}{|c|c|c|c|c|c|c|}
\hline \multirow{2}{*}{$\begin{array}{l}\text { Variáveis/ } \\
\text { categorias }\end{array}$} & \multicolumn{6}{|c|}{ Dimensões } \\
\hline & Pessoal & Interpessoal & Carreira & Estudo & Institucional & QVA-r Total \\
\hline \multicolumn{7}{|l|}{ Sexo } \\
\hline Feminino & 3,4 & 3,8 & 4,3 & 3,7 & 3,4 & 3,7 \\
\hline Masculino & 3,6 & 4,1 & 4,3 & 3,5 & 3,6 & 3,8 \\
\hline \multicolumn{7}{|l|}{ Faixa etária } \\
\hline Até 20 anos & 3,3 & 3,9 & 4,3 & 3,7 & 3,5 & 3,7 \\
\hline De 21 a 25 & 3,6 & 4,0 & 4,3 & 3,6 & 3,5 & 3,8 \\
\hline $\begin{array}{l}\text { Maior que } \\
25 \text { anos }\end{array}$ & 3,4 & 3,2 & 4,3 & 3,3 & 3,4 & 3,5 \\
\hline \multicolumn{7}{|c|}{$\begin{array}{l}\text { Exerce } \\
\text { atividade } \\
\text { remunerada }\end{array}$} \\
\hline Não & 3,5 & 3,9 & 4,3 & 3,6 & 3,5 & 3,8 \\
\hline Sim & 3,3 & 4,1 & 4,2 & 3,3 & 3,5 & 3,7 \\
\hline \multicolumn{7}{|l|}{ Ano } \\
\hline $1^{a}$ série & 3,3 & 3,8 & 4,3 & 3,6 & 3,4 & 3,7 \\
\hline $3^{a}$ série & 3,7 & 4,0 & 4,3 & 3,6 & 3,6 & 3,8 \\
\hline
\end{tabular}

Observamos na tabela 4 a correlação de Spearman entre as 5 dimensões. As maiores correlações positivas foram consideradas de magnitude moderada entre as dimensões estudo e carreira ( $p$-valor $<0.0001$ e $r=0.61$ ). Podemos assim, aferir que os recursos de aprendizagem parecem estar associados as perspectivas de carreira dos estudantes.

Uma outra correlação que merece destaque ocorreu entre as dimensões institucional e pessoal ( $\mathrm{p}$-valor $<0.0001$ e $\mathrm{r}=0.51$ ). Essa correlação positiva também foi considerada de magnitude moderada, evidenciando que os sentimentos e apreciação pela faculdade parecem estar ligados ao bem-estar psicológico dos estudantes.

Tabela 4 - Análise da correlação de Spearman entre as 5 dimensões

\begin{tabular}{llllll}
\hline \multirow{2}{*}{ Variável } & $\left({ }^{*} \mathrm{p}\right)$ & Variável & & & \\
\cline { 3 - 6 } & Estatística & Pessoal & Interpessoal & Carreira & Estudo \\
\hline \multirow{2}{*}{ Interpessoal } & $\mathrm{r}$ & 0,26 & 1 & & \\
& $\mathrm{p}$-valor & $0,0216^{*}$ & 1 & & \\
\hline \multirow{2}{*}{ Carreira } & $\mathrm{r}$ & 0,43 & 0,14 & 1 & \\
& $\mathrm{p}$-valor & $<.0001^{*}$ & 0,2222 & & \\
\multirow{2}{*}{ Estudo } & $\mathrm{r}$ & 0,50 & 0,09 & 0,61 & \multirow{2}{*}{1} \\
& $\mathrm{p}$-valor & $<.0001^{*}$ & 0,4343 & $<.0001^{*}$ & \\
\hline \multirow{2}{*}{ Institucional } & $\mathrm{r}$ & 0,51 & 0,33 & 0,37 & 0,24 \\
& $\mathrm{p}$-valor & $<.0001^{*}$ & $0,0025^{*}$ & $0,0008^{*}$ & $0,0332^{*}$ \\
\hline
\end{tabular}

$* \mathrm{p} \leq 0,05$ 


\section{Discussão}

Os estudantes que ingressam na Universidade encontram inúmeros desafios: pessoais, interpessoais, familiares e institucionais que merecem uma análise atenta pelas autoridades e serviços acadêmicos, a fim de garantir o compromisso na recepção e apoio aos estudantes (ALMEIDA; CRUZ, 2010).

A transição do Ensino Secundário para o Ensino Superior fomenta inúmeras expectativas positivas, mas também uma série de receios e dificuldades (CUNHA; CARRILLO, 2005).

Por isso, a realização desta pesquisa é fundamental no sentido de fornecer dados que possam auxiliar os gestores na condução do curso frente às dificuldades detectadas.

Alguns autores consideram que as experiências vividas pelos estudantes frente a momentos de estresse, angústias, medos e mudanças, estão relacionados ao método de ensino. Principalmente agora, que os currículos são menos sequenciais e menos apoiados em livros guias e/ou manuais, com horários mais flexíveis, onde os professores são tutores e as novas amizades serão construídas em uma base heterogênea de colegas (ALMEIDA; CRUZ, 2010; MAGALHÃES, 2013).

A vivência acadêmica inicia-se com a entrada do estudante na Universidade e requer adaptação, dentre outros aspectos, a um novo modelo de educação que exige maior autonomia por parte dos estudantes na construção do seu conhecimento e formação profissional (SOARES et al., 2014). Na Famema isto está muito presente, já que o modelo adotado não mais possibilita a presença do professor como alguém que transmite conteúdos. Ao contrário, os estudantes, ainda muito jovens, é que assumem grande parcela de responsabilidade na construção do próprio saber.

Em nosso estudo, verificamos que a faixa etária de idade predominante foi de 21 a 25 anos (60\%) o que pode refletir na adaptação a este novo modelo apresentado. Os resultados também assemelham-se aos encontrados na pesquisa de Mognon e Santos (2013), que verificou que a maior parte dos estudantes universitários está na faixa etária de 22 a 25 anos refletindo nos aspectos adaptativos das vivências acadêmicas.

Porta-Nova (2009), em uma pesquisa apontou que quanto maior a idade dos estudantes, maior a probabilidade de alcançar médias superiores nas dimensões pessoais e estudo.

Entretanto, diferindo deste achado, encontramos em nossa pesquisa uma média inferior para essas dimensões na faixa etária acima de 25 anos. 
As maiores médias encontradas foram para a dimensão carreira $(M=4,3)$, no entanto, não houve diferenças entre as faixas etárias.

Isto corrobora o evidenciado por Almeida e Cruz (2010), que encontrou para a faixa acima de 25 anos mais efetividade para a dimensão carreira.

Observamos em nossa pesquisa que a maioria dos estudantes foi do gênero feminino (60\%), enquanto os estudantes do gênero masculino foram de $40 \%$. Alguns autores encontraram resultados similares em seus estudos, referente à distribuição dos gêneros (PORTA-NOVA, 2009; SCHLEICH, 2006).

Essa predominância de estudantes do gênero feminino é explicada pela literatura, que confirma a tendência cada vez mais crescente de mulheres na profissão médica. Isso vem ocorrendo no Brasil e em diversos países, onde a participação feminina é cada vez maior na área médica em decorrência das mudanças culturais e socioeconômicas (ALMEIDA et al., 2006).

De acordo com Machado (1997), as mulheres ocupavam 1\% na área médica, na década de 1940; já na década de 1990 houve um salto expressivo para $50,5 \%$, conforme estudos do autor.

Até pouco tempo, a Medicina era uma prática quase que exclusiva a homens; com o passar do tempo essa prática vem se alterando de modo progressivo e irreversível com a vinda de mulheres a um ambiente que antes era restrito ao universo masculino (MACHADO, 1997).

Interessantemente, nossa pesquisa demonstrou que os estudantes do gênero masculino apresentaram uma tendência de melhor adaptação global ao curso com QVA-r "Total" de 3,8. As estudantes do gênero feminino localizaram-se com QVA-r "Total" de 3,7.

Estes resultados contrariam o que é descrito na literatura, que menciona serem as estudantes do gênero feminino que apresentam maior média do QVA-r "Total" e, portanto, são mais adaptadas globalmente ao Ensino Superior (GRANADO et al., 2005)

Entretanto a pesquisa de Schleich (2006) verificou que o gênero masculino apresenta melhor desempenho nas dimensões carreira e pessoal.

Em nosso estudo encontramos resultados um pouco diferentes, pois os estudantes do gênero masculino tiveram médias superiores em relação ao gênero feminino nas seguintes dimensões: pessoal $(M=3,6)$, interpessoal $(M=4,1)$ e institucional $(\mathrm{M}=3,6)$. $\mathrm{Na}$ dimensão carreira não houve diferenças entre os gêneros com $(M=4,3)$.

Ainda em relação ao gênero dos estudantes, há evidências que as mulheres apresentam médias estatisticamente maiores na dimensão estudo (WILSON et al., 1996). 
Encontramos resultado semelhante, onde as estudantes mulheres apresentam também média superior na dimensão estudo $(\mathrm{M}=3,7)$ em relação aos estudantes homens que obtiveram média $(M=3,5)$. Diante do encontrado, podemos inferir que as estudantes mulheres talvez sejam mais adaptadas à dimensão estudo em comparação aos estudantes homens.

Isto foi também encontrado no trabalho de Cunha (2004), que relatou ser o gênero feminino mais competente e sistemático com os métodos de estudo; são mais organizadas na planificação e no desenvolvimento dos seus trabalhos, tentam desempenhar trabalhos e tarefas que lhes são exigidos e revelam maior assiduidade nas aulas do que os estudantes do sexo masculino.

Em contraposição, o mesmo autor Cunha (2005), em outra pesquisa, verificou que as mulheres estão mais propensas a desenvolver sinais de ansiedade e depressão e experimentam maior sofrimento psicológico do que os estudantes do gênero masculino. Estes apresentam níveis superiores de bem-estar psicológico, bem como físico e emocional, assim como melhor fator adaptativo.

Essa constatação pode estar relacionada com as dificuldades que as estudantes apresentam na adaptação pessoal, devido às extensas expectativas que esse grupo tende a apresentar em relação às dimensões: acadêmica, social e pessoal. A falta de recursos e estratégias pessoais para resolução de problemas associados às elevadas expectativas de vida universitária, poderão promover maior ameaça pessoal, sofrimento psicológico e desilusão às estudantes, ao longo do primeiro ano do Ensino Superior (TEIXEIRA; CASTRO; PICCOLO, 2007).

No que diz respeito à série que os estudantes se encontram, identificamos que os discentes da $3^{\mathrm{a}}$ série do curso Medicina demonstram ser mais adaptados em relação aos estudantes da $1^{\mathrm{a}}$ série, porém na dimensão carreira não houve diferenças.

Conforme menciona a literatura à medida que o estudante vai progredindo no curso, mais adaptado este vai se tornando ao Ensino Superior (GOMES et al., 2009).

Verificou-se que os estudantes de maior idade demonstram mais maturidade psicológica, além de níveis mais elevados de bem-estar psicológico e de equilíbrio emocional; apresentavam métodos mais consistentes de estudo e estratégias mais eficazes; evidenciam melhor gestão do tempo; pareciam apresentar um conhecimento mais sólido em relação ao curso; e mostraram-se melhores preparados para os exames e para o aproveitamento dos recursos de aprendizagem disponibilizados pela Universidade, em relação aos estudantes com menos idade (GOMES et al., 2009). 
De acordo com Fernandes e Almeida (2005), confirmam-se estes resultados, uma vez que nas suas investigações revelou-se que estudantes com mais idade apresentam maiores competências e melhores estratégias de estudos, maior compromisso, satisfação e conhecimento com o curso.

De acordo com Botto (2013) e Salgado et al. (2010), há relação entre a idade e fator adaptativo dos estudantes, onde os autores referenciam que os estudantes com mais idade apresentam melhores níveis de adaptação nas dimensões, pessoal, acadêmico (curso), carreira, institucional, em relação ao discentes mais novos.

Corroboram com os resultados os estudos de Freitas, Raposo e Almeida (2007), onde se averiguou que os estudantes com maior idade demonstravam níveis superiores de adaptação na esfera institucional e carreira do Ensino Superior.

Em relação aos estudantes que exercem algum tipo de atividade remunerada mesmo sem período e horário fixo, constatamos uma média inferior no QVA-r "Total" $(\mathrm{M}=3,7)$, em relação aos estudantes que apenas estudam QVA-r "Total" $(\mathrm{M}=3,8)$. Concluímos que o fato de exercer atividade remunerada concomitante ao estudo pode interferir na adaptação ao Ensino Superior e consequentemente no processo adaptativo ao estudo. Isso confirma as médias inferiores na dimensão estudo $(\mathrm{M}=3,3)$ pelos estudantes que exerciam atividades remuneradas, em relação aos que não exerciam nenhuma atividade remunerada $(M=3,6)$.

Interessantemente, sabemos não ser usual encontrar estudantes de Medicina em tempo integral com algum tipo de atividade de trabalho remunerado.

De acordo com Fernandes e Almeida (2005), os estudantes que manifestaram os piores rendimentos universitários estavam envolvidos com algum tipo de atividade extraclasse, ocasionando assim um distanciamento das atividades acadêmicas, o que apontava para um menor interesse nas atividades curriculares.

No entanto, os estudantes que exercem somente atividade de cunho acadêmico apresentam melhor rendimento no curso e consequentemente maior nível de aprendizagem (ALMEIDA et al., 2007).

Percebe-se que os estudantes que são apoiados emocionalmente por suas famílias, sentem este apoio durante toda a vida, o que contribui na capacidade de enfrentar a ansiedade envolvida na separação do ambiente familiar e no estabelecimento de novas relações que se caracterizam no contexto universitário ao o favorecimento de maior integração.

Encontramos médias elevadas na dimensão carreira e estas podem significar não uma satisfação com a escolha baseada em critérios realistas, e sim uma expectativa positiva frente ao que está por vir, mas que ainda não foi experimentado de fato. Sendo assim, é compreensível que a satisfação com a escolha 
decline com o passar do tempo, na medida em que as limitações do curso e da profissão vão se tornando aparentes e as frustrações podem surgir.

Há diversos estudos que têm utilizado o QVA-r, demonstrando várias correlações relevantes de foro psicológico. Neste sentido, observamos como o método empregado pela Instituição de Ensino pode contribuir de forma positiva ou negativa na vida acadêmica dos discentes.

\section{Conclusão}

Evidenciamos que os estudantes do sexo masculino revelam uma melhor adaptação global, como exceção da dimensão estudo onde as discentes do sexo feminino demostraram melhor adaptação frente a currículos inovadores.

Constata-se, também, que o fator idade, pode ser um fator aliado na adaptação do estudante ao Ensino Superior, sendo que quanto mais maduro o estudante, maiores são as possibilidades de adaptação aos contextos adaptativos.

Verificamos também, que a $3^{\text {a }}$ série encontram-se mais adaptada com a instituição de ensino e consequentemente ao método de ensino empregado pela mesma, em relação aos estudantes da $1^{\mathrm{a}}$ série inferindo que com o passar dos anos a adaptação torna-se mais efetiva.

A dimensão carreira apresenta-se bastante presente nas vivências acadêmicas para ambas as séries, ressaltando que essa dimensão está relacionada ao sentimento com o curso frequentado e perspectivas de carreira, incluindo a satisfação com o curso e percepção de competências para o mesmo.

Esta pesquisa abre possibilidades para futuras investigações que possam explorar mais o tema, como condição de ampliação do conhecimento, contribuindo assim para melhor compreensão, tanto do próprio estudante como também de características ligadas às instituições de ensino superior.

\section{Referências}

ALMEIDA, Leandro Santos et al. Rendimento académico no ensino superior: estudo com alunos do $1^{\circ}$ ano. Revista Galego-Portuguesa de Psicoloxía e Educación, Coruña, v. 14, n. 1, p. 207-220, 2007.

ALMEIDA, Leandro Santos et al. Acesso e sucesso no ensino superior em Portugal: questões de género, origem sócio-cultural e percurso académico dos alunos. Psicologia: Reflexão e Crítica, Porto Alegre, v. 19, n. 3, p. 507514, 2006. 
ALMEIDA, Leandro Santos; SOARES, Ana Paula C.; FERREIRA, Joaquim Armando. Questionário de vivências acadêmicas (QVA-r): Avaliação do ajustamento dos estudantes universitários. Avaliação Psicológica, Coimbra, v. 1, n. 2, p. 81-93, 2002.

ALMEIDA, Leonardo Santos et al. Percursos e expectativas de estudantes universitários: estudo com alunos do $1^{\circ}$ ano da Universidade do Minho. In: MEDEIROS, Teresa; PEIXOTO, Ermelindo (Org.). Actas do Congresso Internacional Desenvolvimento e Aprendizagem: do ensino secundário ao ensino superior. Ponta Delgada: Universidade do Açores, 2005. p. 237-251. Disponível em: <http://repositorium.sdum.uminho.pt/ handle/1822/12060>. Acesso em: 25 jan. 2015.

ALMEIDA, Leonardo Santos. Transição, adaptação académica e êxito escolar no ensino superior. Revista Galego-portuga de Psicoloxía e Educación, Coruña, v. 14, n. 2, p. 203-215, 2007.

ALMEIDA, Leonardo Santos; CRUZ, José Fernando A. Transição e adaptação académica: reflexões em torno dos alunos do $1^{\circ}$ ano da Universidade do Minho. Comunicação apresentada no Congresso Ibérico Ensino Superior em Mudança: tensões e possibilidades. Braga, 2010. Disponível em: $<$ http://repositorium.sdum.uminho.pt/handle/1822/11543>. Acesso em: 15 mar. 2015.

BOTTO, Sotero Filipe Vaz Donas. Vinculação em jovens adultos: processo de individuação em contexto universitário. Dissertação (Mestrado) 2013. 253 f. Vila Real (PT): Universidade de Trás-os-Montes e Alto Douro, 2013.

BRASIL. Ministério da Educação. Conselho Nacional de Educação. Câmara de Educação superior. Resolução no 3, de 20 de junho de 2014. Institui as Diretrizes Curriculares Nacionais do Curso de Graduação em Medicina e da outras providências. Diário Oficial da União, Brasília, DF, 23 jun. 2014. Seção1, p. 8.

CUNHA, Simone Miguez. A inteligência e as habilidades sociais na adaptação de alunos ao curso superior: um estudo com alunos do $1^{\circ}$ ano do Instituto Militar de Engenharia. 2004. Dissertação (Mestrado) Universidade Gama Filho, Rio de Janeiro, 2004.

CUNHA, Simone Miguez; CARRILLO, Denise Madruga. O processo de 
adaptação ao ensino superior e o rendimento acadêmico. Psicologia Escolar e Educacional, Campinas, v. 9, n. 2, p. 215-224, 2005.

DANCEY, Christine P.; REIDY, John. Estatística sem matemática para psicologia: usando SPSS para Windows. Porto Alegre: Artmed, 2006.

FACULDADE DE MEDICINA DE MARÍLIA. Necessidades de Saúde 3 e Prática Profissional 3: $3^{\text {a }}$ série do Curso de Medicina. Marília: Famema, 2012.

FELDT, Ronald C et al. Personality, career indecision, and college adjustment in the first semester. Individual Differences Research, Pittsburgh, v. 9, n. 2, p.107-114, jun. 2011.

FERNANDES, Eugénia; ALMEIDA, Leandro Santos. Expectativas e vivências acadêmicas: impacto no rendimento dos alunos do $1 .^{\circ}$ ano. Psychologica, Coimbra, v. 40, p. 267-278, 2005.

FEUERWERKER, Laura Camargo Macruz; LIMA, Valéria Vernaschi. Os paradigmas da atenção à saúde e a formação de recursos humanos. Brasília: Ministério da Saúde, 2002.

FREITAS, Helena Cristina das Neves Mira; RAPOSO, Nicolau de Almeida Vasconcelos; ALMEIDA, Leandro Santos. Adaptação do estudante ao ensino superior e rendimento acadêmico. Revista Portuguesa de Pedagogia, Coimbra, v. 41, n. 1, p. 179-188, 2007.

FRIEDLANDER, Laura J. et al. Social support, selfes teem and stress as predictors of adjustment to university among first-year undergraduates. Journal of College Student Development, Alexandria, v. 48, n. 3, p. 259274, May/June 2007.

GOMES, Andréia Patrícia; REGO, Sergio. Transformação da educação médica: é possível formar um novo médico a partir de mudanças no método de ensino-aprendizagem?. Revista Brasileira de Educação Médica, Rio de Janeiro, v. 35, n. 4, p. 557-566, out./dez. 2011.

GOMES, Romeu et al. Aprendizagem Baseada em Problemas na formação médica e o currículo tradicional de medicina: uma revisão bibliográfica. Revista Brasileira de Educação Médica, Rio de Janeiro, v. 33, n. 3, p. 433440, jul./set. 2009. 
GRANADO, José Inácio Fernandes et al. Integração académica de estudantes universitários: contributos para a adaptação e validação do QVA-r no Brasil. Psicologia e Educação, Covilhã, v. 2, n. 4, p. 31-41, 2005.

MACHADO, Maria Helena. As médicas no Brasil. In: MACHADO, Maria Helena, coordenadora. Os médicos no Brasil: um retrato da realidade. Rio de Janeiro: Fiocruz, 1997. p. 147-161.

MAGALHÃES, Mauro de Oliveira. Sucesso e fracasso na integração do estudante à universidade: um estudo comparativo. Revista Brasileira de Orientação Profissional, São Paulo, v. 14, n. 2, p. 215-226, jul./dez. 2013.

MAZÉ, Corinne; VERLHIAC, Jean-François. Stress and coping strategies of first-year students: distinctive roles of transactional and dispositional factors. Psychologie Française, Paris, v. 58, n. 2, p. 89-105, jun. 2013.

MOGNON, Jocemara Ferreira; SANTOS, Acácia Aparecida Angeli dos. Relação entre vivência acadêmica e os indicadores de desenvolvimento de carreira em universitários. Revista Brasileira de Orientação Profissional, São Paulo, v. 14, n. 2, p. 227-237, dez. 2013.

PORTA-NOVA, Rui Manuel Marques do Monte. Adaptabilidade, competências pessoais e bem-estar psicológico de jovens do ensino superior na área das ciências da saúde. 2009. 267 f. Tese (Doutorado) Instituto de Ciências Biomédicas Abel Salazar, Porto, PT, 2009.

SALGADO, A. et al. O Problem-based learning e suas implicações nas expectativas e vivências académicas dos estudantes do $1^{\circ}$ ano de Cursos de Tecnologias da Saúde. In: NOGUEIRA, C et al. (Ed.). Actas do VII Simpósio Nacional de Investigação em Psicologia. Braga: Universidade do Minho, 2010. p. 2839-2853.

SANTOS, Luísa; ALMEIDA, Leandro Santos. Vivências académicas e rendimento escolar: estudo com alunos universitários do $1^{\circ}$ ano. Análise Psicológica, Lisboa, v. 19, n. 2, p. 205-217, 2001.

SARRIERA, Jorge Castellá et al. Estudo comparativo da integração ao contexto universitário entre estudantes de diferentes instituições. Revista Brasileira de Orientação Profissional, São Paulo, v. 13, n. 2, p.163-172, dez. 2012. 
SCHLEICH, Ana Lucia Righi. Integração na educação superior e satisfação acadêmica de estudantes ingressantes e concluintes: um estudo sobre relações. 2006. 186 f. Dissertação (Mestrado) - Universidade Estadual de Campinas, Campinas, 2006.

SOARES, Adriana Benevides et al. O impacto das expectativas na adaptação acadêmica dos estudantes no Ensino Superior. Psico USF, Bragança Paulista, v. 19, n. 1, p. 49-60, jan./abr. 2014.

SOARES, Adriana Benevides; POUBEL, Lincoln Nunes; MELLO, Thatiana Valory dos Santos. Habilidades sociais e adaptação acadêmica: um estudo comparativo em instituições de ensino público e privado. Aletheia, Canoas, n. 29, p. 27-42, jun. 2009.

\section{STELLA, Regina Ceres de Rosa. A prática médica no contexto das} Diretrizes Curriculares Nacionais para o Curso de Medicina. São Paulo: [s.n.], 2007.

TEIXEIRA, Marco Antônio Pereira; CASTRO, Graciele Dotto; PICCOLO, Luciane da Rosa. Adaptação à universidade em estudantes universitários: um estudo correlacional. Interação em Psicologia, Curitiba, v. 11, n. 2, p. 211220, 2007.

VILLAR, Jorge Daniel. Adaptação de questionário de vivência universitária com estudantes de Arquitetura e de Engenharia. 2003. 142 f. Dissertação (Mestrado), Universidade São Francisco, Itatiba, 2003.

WILSON, John F. et al. Student's Quality of life After a Major Curriculum Change. Academic Medicine, Philadelphia, v. 71, n. 10, p. 40-42, 1996.

Diego Roberto Lima dos Anjos - Faculdade de Medicina de Marília Marília | SP | Brasil. Contato: diegolimafisio@hotmail.com

Rinaldo Henrique Aguilar-da-Silva - Faculdade de Medicina de Marília Marília | SP | Brasil. Contato: aguilar@famema.br 
